\title{
Quantum Dot Quantum Cascade Detector on Si Substrate
}

\author{
Jian Huang, ${ }^{1, a)}$ Daqian Guo, ${ }^{2, \text { a) }}$ Zhuo Deng, ${ }^{1}$ Wei Chen, ${ }^{1}$ Tinghui Wu, ${ }^{1}$ Yaojiang Chen, ${ }^{1}$ Huiyun Liu ${ }^{2}$, Jiang \\ $\mathrm{Wu}^{* 2}$, Baile Chen ${ }^{* 1}$ \\ ${ }^{1}$ Optoelectronic Device Laboratory, School of Information Science and Technology, ShanghaiTech University, Shanghai 201203, China \\ ${ }^{2}$ Department of Electronic and Electrical Engineering, University College London, London WC1E 7JE, United Kingdom \\ a) Jian Huang and Daqian Guo contributed equally to this work. \\ E-mail address:jiang.wu@ucl.ac.uk; chenbl@shanghaitech.edu.cn.
}

\begin{abstract}
We demonstrate a quantum cascade detector monolithic grown on Si substrate for mid infrared application. Sub-monolayer dots were used as absorption region for normal incidence light detection around $6 \mu \mathrm{m}$.

OCIS codes: (250.0040) Detectors; (040.5160) Photodetectors; (250.5590) Quantum-well, -wire and -dot devices; (040.3060) Infrared
\end{abstract}

\section{Introduction}

Mid infrared photodetectors have important applications in gas monitor, thermal imaging and free space communication. Quantum well infrared photodetector (QWIP) shows high responsivity, and QWIP focal plane arrays have been demonstrated for infrared imaging applications[1]. However, QWIP under photoconductive (PC) mode show high dark current, which considerably reduces the detectivity. Therefore, photovoltaic (PV) QWIP is desirable with excellent Johnson noise limited detectivity. Quantum cascade detectors (QCD) is a type of PV intersubband photodetectors, which recently attracted significant interests [2]. QCD based on quantum well have been demonstrated in short infrared, mid infrared and long infrared wavelength ranges. However, the quantum well QCD don't have normal incidence light response due to the inter-subband transition selection rules of quantum wells. Initially, the quantum cascade detectors with sub-monolayer quantum dots layer was demonstrated on GaAs substrate by our group, which shows normal incident light response.

In this paper, we report a quantum cascade detectors monolithic grown on Si substrate based on sub-monolayer quantum dots, which has potentials to directly integrate the III-V materials with CMOS for MWIR application.

\section{Device Structures}

The device structure is shown in Fig 1. The buffer layers of AlGaAs/GaAs materials were firstly grown on n-doped silicon substrate with $4^{\circ}$ offcut. Then, the defect filter layers made up of InGaAs/GaAs superlattices were grown. Sequentially, the quantum cascade detector structure with sub-monolayer QDs layers was grown for mid infrared detections.

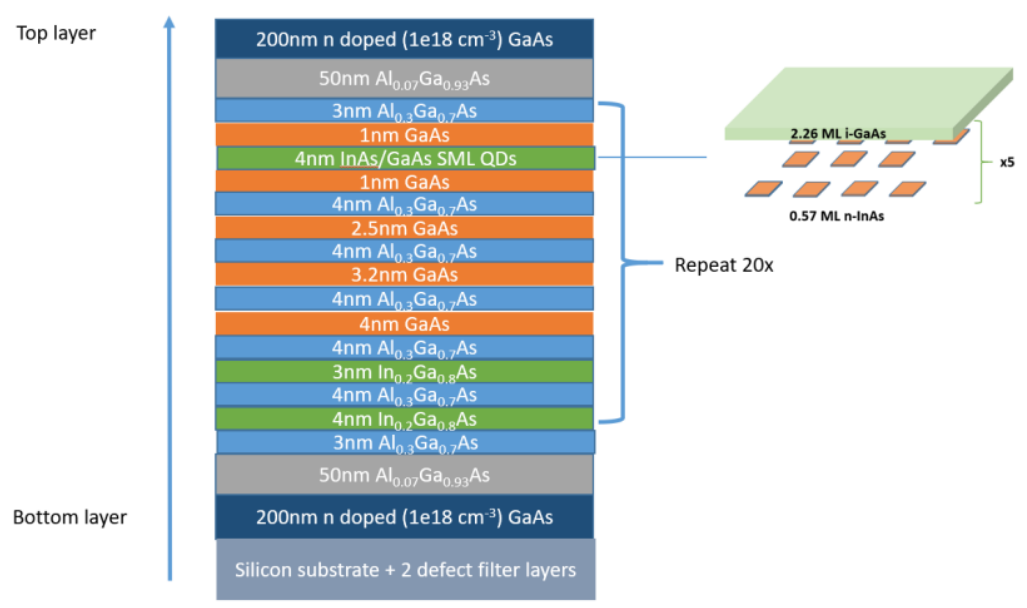

FIG. 1. Schematics of the device structure.

After the material growth, the sample was fabricated into a set of unpassivated mesa-isolated test structures with device diameters ranging from $20 \mu \mathrm{m}$ to $500 \mu \mathrm{m}$ using standard UV photo-lithographic processing technique. Wet chemical etching process $\left(\mathrm{H}_{3} \mathrm{PO}_{4}: \mathrm{H}_{2} \mathrm{O}_{2}: \mathrm{H}_{2} \mathrm{O}=1: 1: 8\right)$ was used to define the mesa structures. $50 \mathrm{~nm} / 300 \mathrm{~nm}$ Ti/Au were deposited by e-beam 
evaporator as the top and bottom $\mathrm{n}^{+}-$GaAs metal contact layers.

\section{Results}

The dark current of the Si based QCD devices at $77 \mathrm{~K}$ were measured in low temperature probe station by semiconductor device analyzer. The devices show dark current density of $2.1 \times 10^{-8} \mathrm{~A} / \mathrm{cm}^{2}$, the calculated resistancearea product $\left(\mathrm{R}_{0} \mathrm{~A}\right)$ is $1.13 \times 10^{7} \Omega . \mathrm{cm}^{2}$. These performance are comparable to the other QCD grown on native III-V substrate[3].

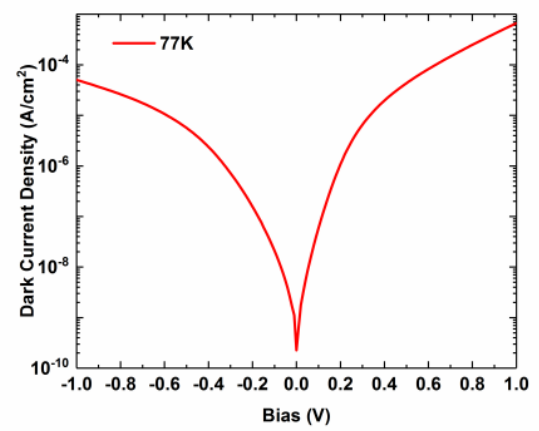

Fig 2. Dark current density of Si based QCD device at $77 \mathrm{~K}$

The normal incident optical response of the Si QCD was measured by Fourier transform infrared spectrometer, and calibrated by a blackbody resource at $700^{\circ} \mathrm{C}$ under zero bias as shown in Fig 3(a). The John noise limit detectivity of the QCD is calculated in Fig 3(b). The device show high detectivity of $3 \times 10^{10} \mathrm{~cm} \cdot \mathrm{Hz}^{1 / 2} / \mathrm{W}$ under zero bias at $6.2 \mu \mathrm{m}$ at $77 \mathrm{~K}$.

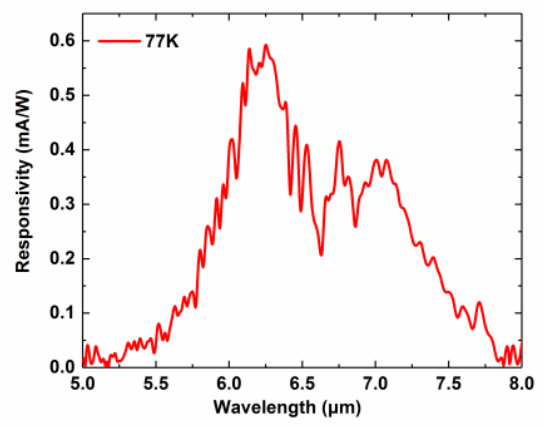

(a)

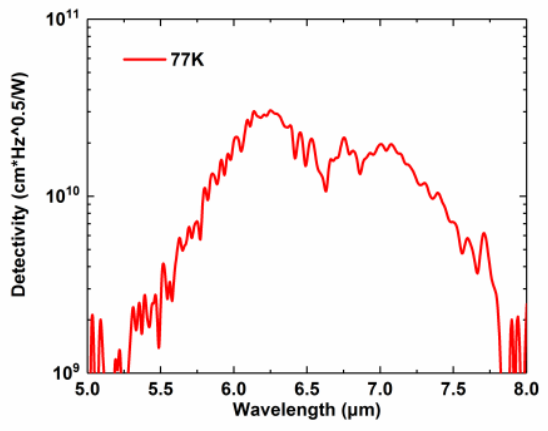

(b)

Fig 3 (a) Optics response of the QD-QCD measured at temperature of $77 \mathrm{~K}$ (b) The Johnson noise limited detectivity of the QDQCD at $77 \mathrm{~K}$.

\section{Summary}

In summary, we demonstrate a sub-monolayer QD QCD on Silicon substrate. The preliminary results show that the performance of the QCD on Silicon is comparable to the other QCD on native III-V semiconductor substrates. It is very promising for future silicon photonics integration application.

\section{Reference:}

[1] B. Levine, "Quantum - well infrared photodetectors," Journal of applied physics, vol. 74, pp. R1-R81, 1993.

[2] F. R. Giorgetta, E. Baumann, M. Graf, Q. Yang, C. Manz, K. Kohler, et al., "Quantum cascade detectors," IEEE Journal of Quantum Electronics, vol. 45, pp. 1039-1052, 2009.

[3] A. Barve and S. Krishna, "Photovoltaic quantum dot quantum cascade infrared photodetector," Applied Physics Letters, vol. 100, p. 021105, 2012. 